This is the peer-reviewed but unedited manuscript version of the following article: Watson, R., Bagnasco, A. Catania, G., Aleo, G., Zanini, M., \& Sasso, L. (2017). The Edinburgh Feeding Evaluation in Dementia Scale: A Longitudinal Study in Nursing Home Residents. Dementia and Geriatric Cognitive Disorders, 44(3-4), (196-202). The final, published version is available at http://www.karger.com/10.1159/000478519.

The Edinburgh Feeding Evaluation in Dementia Scale: A longitudinal study in nursing

\title{
home residents
}

Short title: The EdFED-I Scale: A longitudinal study

Roger WATSON, PhD, RN, FRCN, FAAN

Professor of Nursing

Faculty of Health and Social Care

University of Hull,

UK, HU6 7RX

Tel. +441482464625

Fax +44 1482464695

Email: R.Watson@hull.ac.uk

Annamaria BAGNASCO, PhD, MEdSc, MSN, RN

Assistant Professor of Nursing \& Education Coordinator,

Department of Health Sciences,

University of Genoa,

Via Pastore, 1

16132 Genoa, Italy

Tel. 00390103538415

Fax 00390103538552

Email: annamaria.bagnasco@unige.it

Gianluca CATANIA, PhD, MSN, RN

Research Fellow \& Lecturer

Department of Health Sciences

University of Genoa,

Via Pastore, 1

16132 Genoa, Italy

Tel. 00390103538519

Fax 00390103538552

Email: gianluca.catania@edu.unige.it

Giuseppe ALEO, PhD, MA

Research Fellow \& Lecturer

Department of Health Sciences

University of Genoa,

Via Pastore, 1

16132 Genoa, Italy

Tel. 00390103538519

Fax 00390103538552

Email: giuseppe.aleo@edu.unige.it 
Milko ZANINI, PhD, MSN, MSoc, RN

Research Fellow \& Lecturer

Department of Health Sciences

University of Genoa,

Via Pastore, 1

16132 Genoa, Italy

Tel. 00390103538519

Fax 00390103538552

Email: milko.zanini@edu.unige.it

Loredana SASSO, MEdSc, MSN, RN, FAAN

Associate Professor of Nursing

Department of Health Sciences

University of Genoa,

Via Pastore, 1

16132 Genoa, Italy

Tel. 00390103538513

Fax 00390103538552

Email: l.sasso@unige.it

\section{Corresponding author}

Annamaria BAGNASCO, PhD, MEdSc, MSN, RN

Assistant Professor of Nursing \& Education Coordinator,

Department of Health Sciences,

University of Genoa,

Via Pastore, 1

16132 Genoa, Italy

Tel. 00390103538415

Fax 00390103538552

Email: annamaria.bagnasco@unige.it

\section{Keywords}

Dementia, Feeding, Item Response Theory, Mokken Scaling, EdFED

\section{Abstract}

Aims/Background: The Edinburgh Feeding Evaluation in Dementia (EdFED) scale has been shown to have good psychometric properties using a range of methods including Mokken 
scaling. We aimed to study the Italian version of the EdFED using Mokken scaling. Methods: Data were gathered at seven time points from 401 nursing home residents affected by dementia in the course of a six-month intervention study and analysed using analysis of variance, Mokken scaling and person-item fit statistics. Results: The properties of the EdFED-I scale were stable over the course of the study with four items showing invariant items ordering at all time points. Some items behaved differently at different levels of difficulty in the scale and also depending on the mean level of feeding difficulty. The test information function showed a dip in the mid-range of difficulty scores.

\section{INTRODUCTION}

Dementia is characterised by loss of memory and decline in cognitive function [1]. There are many causes of dementia but most commonly dementia is associated with Alzheimer's disease, Lewy body dementia and vascular dementia [2]. In addition to the psychological effects listed above, dementia leads to decline in activities of daily living and the ability to self-care [3]. In the advanced stages of dementia it is very common for there to be a marked decline in the ability to feed self [4]. Therefore, we need to be able to measure feeding difficulty and to characterise the stages in feeding difficulty so that appropriate interventions can be applied.

The Edinburgh Feeding Evaluation in Dementia (EdFED) scale [5] remains the only validated instrument internationally [6] for assessing feeding difficulty in dementia. The EdFED scale measures difficulty with feeding in older people with dementia including nursing actions related to difficulty with feeding (2 items, e.g. supervision), indicators of difficulty with feeding (2n items, e.g. spillage) and behavioural aspects of difficulty with 
feeding (6 items, e.g. spitting out food). A hierarchy of feeding difficulty was observed [7], which was confirmed using Mokken scaling [8] and which is reproducible [9].

The EdFED has low to moderate but statistically significant correlations with common indicators of nutritional status: body mass index; body weight; time taken to eat; the Barthel ADL scale; and Mini Mental State Examination [10,11]. Therefore, the EdFED scale is a valid measure of feeding difficulty in dementia. There is a cluster of behavioural items forming a hierarchy. However, we need to know more about the EdFED scale; for example, how items behave in lower and higher scoring patients and if the hierarchy of items are sustained across time.

\section{The present study}

A large longitudinal intervention study on nutrition in older people in nursing homes in Italy (Zanini M, Bagnasco A, Catania G, et al. JAMDA unpublished) using a modified texture diet (The Nutricare Project) provided a large sample of mainly older people with dementia. An outcome measure used in the study was the EdFED-I scale [11]. The aim of the present study was to study the properties of the EdFED-I scale over time and across a range of the latent trait using IRT methods, including Mokken scaling. The research question guiding the study was: how stable is the EdFED-I scale as a measure of feeding difficulty in older people with dementia? The criterion for judging stability across time was the ordering of items by mean score; in a very stable scale this would be similar at each time point but in one that is unstable the item ordering would differ between time points. This is an important property to investigate as, if the ordering of the items is stable then the overall feeding difficulty can be related to the scores on specific items. If the ordering of the items does not change across time then a meaningful comparison can be made between total scores on the scale at each 
time and this increases its utility as a measure of change in feeding difficulty either due to deterioration of the patient of due to an intervention.

\section{Mokken scaling}

Mokken scaling analysis (MSA) is a non-parametric method item response theory (IRT) method that includes Rasch scaling [13]. A non-technical explanation of the method is provided elsewhere [14]. but the basic premise of IRT is that there is a stochastic relationship between the score on an item in a questionnaire and the score on the latent trait that is being measured; this relationship is described as the item characteristic curve (ICC) which is the unit of analysis in IRT methods, including MSA. This relationship-for items that conform to an IRT model-imposes a hierarchy of items along the continuum of the latent trait in terms of 'difficulty', which in IRT terms means the likelihood with which items are endorsed by respondents.

Item quality in IRT is judged by the extent to which item scores continually increase as the latent trait increases (monotonicity) and the extent to which they do no intersect with other items along the latent trait (invariant item ordering; IIO), which is stronger if items are well spaced along the latent trait [14]. Other aspects of MSA follow, including the probability of finding a Mokken scale in a dataset and the reliability of derived scales. MSA is nonparametric as it does not predict a precise relationship between the score on an item and the latent trait, merely that they are monotonic and non-intersecting [15].

\section{DESIGN AND METHODS}

A longitudinal survey; data were gathered using the EdFED-I scale by nurses working with 401 older people in 21 nursing homes in Northern Italy between January 2014 and June 2015. 
Data were gathered monthly from baseline to 6 months. Participants included mainly older people with mild to severe dementia including 10 participants without dementia. Mean age of participants was 80 years (SD12); 66 (16.5\%) were male and 333 (83\%) were female with $0.5 \%$ missing data for gender.

\section{The EdFED scale}

The version of the EdFED scale used in this study contains 10 items and these are rated by observers - usually nurses or carers who are familiar with the older person-on a three-point scale from 0 to 2 ('never', 'sometimes', and 'often'). The EdFED-I scale was translated from English into Italian as previously described [11].

\section{Analysis}

Data were saved in a tab-delimited form suitable for item analysis using TestGraf [16] and also imported into package 'foreign' in $R$ and converted to a format suitable for analysis in $R$ using the package 'mokken' [17]. Data at baseline and 6 months were analysed using the automated item selection procedure (aisp) and analysed for violations of monotonicity, reliability. The standard errors of the items and the items pairs were used to calculate 95\% confidence intervals [18]. ICC pair plots were generated and inspected for intersecting items and outlying items. TestGraf was used to generate option characteristic curves (OCCs) which relate the probability of the score on an option (i.e. 'never'; 'sometimes'; 'often') to the expected score on the latent trait—and test information functions (TIFs) which plot of the reciprocal of the variance in the item scores at any point along the latent trait [19].

\section{Ethics}


The study was approved by the Ethics Committee of the University of Genoa. Family members signed a consent form to allow data collection from participants.

\section{RESULTS}

Demographics of the participants are provided above. Baseline data were used to assess the quality of the EdFED-I scale items using ICC pair plots (see Supplementary file 1 for all of the ICC pair plots) and showed that one item (item 5: 'Does the patient ever refuse to eat?') was not monotonic (Supplementary file 1 Figure 1) and that one item (item 3: 'Is there spillage while feeding?') intersected with other items (Supplementary file 1 Figure 2). Items 1 and 2 ('Does the patient require close supervision while feeding?' and 'Does the patient require physical help with feeding?', respectively) lay very close together, showed a ceiling effect and were positioned far from the other items (Supplementary file 1 Figure 3). Supplementary Figures 4a-f show six ICC pairs from four items that do not intersect and probably show strong IIO (Item 7: ‘Does the patient ever refuse to open his/her mouth?’, Item 8: 'Does the patient spit out his/her food?', Item 9: 'Does the patient leave his/her mouth open allowing food to drop out?’, Item 10: ‘Does the patient refuse to swallow?’).

The aisp consistently showed at baseline and 6 months that Item 5 ('Does the patient ever refuse to eat?') did not scale, except at time waves 6 and 7 where item 7 ('Does the patient ever refuse to open his/her mouth?') did not scale. All the remaining items at all time waves formed a single Mokken scale with Loevinger's coefficient $(\mathrm{Hs}$ ) ranging from 0.46 to 0.48 indicating a moderately strong scale.

IIO was investigated in the total set of items and to demonstrate the process the results from baseline are shown in Table 1 (all time wave data available in Supplementary file 2). Item 3 
('Is there spillage while feeding?') and item 5 ('Does the patient ever refuse to eat?') are removed at steps 1 and 2 of the IIO investigating process and, after five steps, six items remain. Table 2 shows all the EdFED-I scale items ordered by their mean values at baseline and 6 months along with the Loevinger's coefficients $(H)$ for all items (in brackets) and scale $H$ and Htrans $\left(H^{T}\right)$, which is a measure of the strength of IIO. The scale is reliable as shown by Rho exceeding 0.70 at both points. Rho is a measure of reliability generated by the Mokken scaling package and which is analogous to Cronbach's alpha but less sensitive to the number of items in the scale. $H^{T}$ ranges from 0.53 to 0.58 indicating strong IIO for items 7,8 , 9, and 10 (respectively: 'Does the patient ever refuse to open his/her mouth?'; 'Does the patient spit out his/her food?'; 'Does the patient leave his/her mouth open allowing food to drop out?'; 'Does the patient refuse to swallow?'). IIO for the total scale in Table 2 is acceptable but lower than that established in Table 1 where the scale was reduced only to those items at $\mathrm{T} 1$ showing zero violations of IIO. This pattern of lower IIO for the total scale compared with those items only showing IIO (Supplementary file 2) is inevitable; the greater the number of items analysed, the greater the likelihood that some will violate IIO. Ordered by mean item score the Mokken scales of all items are consistently anchored by items 1 and 9 ('Does the patient require close supervision while feeding?' and 'Does the patient leave his/her mouth open allowing food to drop out?', respectively with item 9 indicating the highest level of feeding difficulty measured by the EdFED-I scale. Otherwise the ordering of items is consistent apart from an anomaly between items 3 and 7 ('Is there spillage while feeding?' and 'Does the patient ever refuse to open his/her mouth?', respectively) and one involving item 6 ('Does the patient turn his/her head away while being fed?'). It should be noted, however, the 95\% confidence intervals for items 3 and 5 ('Is there spillage while feeding?' and 'Does the patient ever refuse to eat?', respectively) include the lowerbound value (0.30) of Loevinger's coefficient for items $(H s)$. 
Supplementary file 1 Figures 5a-6 and 6a-d show a selection of OCCs compared across the study at time waves 1 and 7. Specifically, items 1 and 2 ('Does the patient require close supervision while feeding?' and 'Does the patient require physical help with feeding?', respectively) and items 6 and 9 ('Does the patient turn his/her head away while being fed?' and 'Does the patient leave his/her mouth open allowing food to drop out?', respectively) are presented. Items 1 and 2 show a ceiling effect at time wave 1 but this effect is not apparent in item 2 at time wave 7. There is little change in the OCC of items 6 and 9 between time waves 1 and 2.

Supplementary file 1 Figures 7 and 8 show the test information functions at time waves 1 and 7, respectively. At both time waves the level of information in the scale is inconsistent across the score range of the EdFED-I scale. The level of information is highest in the higher range of scores at time wave 1 and in the lower range of time wave 7; at both time waves there is a dip in the information in the mid-region of the range

\section{DISCUSSION}

This study extends previous work on an Italian translation of the EdFED scale- the EdFED-I scale-making use of a larger sample with data sampled periodically to address the question of whether or not the EdFED scale was stable across time. The IRT properties of the EDFEDI scale were also studied in more detail.

Existing work on the EdFED scale was largely supported in that a hierarchy of items was observed among the behavioural items in the EdFED-I scale and the remaining items did not cluster with these. This suggest that, in common with other versions of the EdFED scale, the 
EdFED-I scale contains two sets of items identifiable by Mokken scaling: those concerned with the indicators and feeding difficulty and nursing interventions and those related to the feeding related behaviours in dementia. In common with the previous study [11] the items related to nursing interventions score highest and show a ceiling effect. However, unlike the previous study — where the indicators of feeding difficulty also scored high and higher than all of the behavioural items - in the present study the item related to spillage (item 3) shows a floor effect and the item related to leaving food on the plate (item 4) scores in the middle region. These observations related to the indicator items suggest that the population studied here differed in severity from the previous study - and the scores on the behavioural items suggest that this is the case. It is also possible that the care environment differed or that care staff were more aware of spillage and that adopted cutlery and crockery were being used; it is certainly the case that the food used in the present study—as part of the intervention—was specifically textured and packaged. It is also apparent that, while the item on leaving food on the plate (item 4) did not scale, it did show a steady decline over the study suggesting that this is responding to the intervention in the study.

With one exception, the Mokken scaling properties of the items were the same at baseline and 6 months. The item about spillage (item 3), while scoring very low, also had very wide confidence intervals and the item enquiring about general refusal to eat (iem5) intersected with other items. The latter observation is not surprising as, being very general and nonspecific, this item may be harder to score accurately. While the item about turning the head away (item 6) scaled well it did not show IIO, unlike the four remaining items, which scored invariantly across the whole study. This invariance in score ordering reflects previous studies [10,11]—while differing slightly from the previous EdFED-I scale study [11]—and shows that leaving food in the mouth and allowing it to fall out (item 9) is the most difficult item in 
the EdFED-I scale, in common with the original EdFED scale [11] suggesting that this indicates a very serious level of difficulty with feeding. The utility of these observations will be discussed below.

This is the first study where the items of the EdFED scale have been inspected in more detail, specifically, at the level of item options. The behaviour of the item option curves selected here needs to be interpreted against the total EdFED-I scale score across the study, which declines significantly from the start to the end of the study, indicating a decline in feeding difficulty. Item 1 - related to supervision - behaves similarly at times 1 and 7 (which represent relatively high and low feeding difficulty, respectively) in that it is only responsive in the lower range of feeding difficulty and shows a ceiling effect at a total score of approximately 9 regardless of the mean level of feeding difficulty. Item 2 - related to physical help - is responsive across the whole range of the latent trait at time 7 but shows a ceiling effect at 1 time indicating that it behaves differently depending on the mean level of feeding difficulty. Items 6 and 9-related to turning the head away and allowing food to fall of the mouth, respectively_-both behave similarly at times 1 and 7 showing floor effects up to the mid-range of the total score and are both more responsive at higher levels of the latent trait but do not behave differently depending on the mean level of feeding difficulty. Therefore, without inspecting each item at each time, it is clear that different items are responsive at different levels of the latent trait and that some may behave differently depending on the mean level of feeding difficulty. The two information function plots support this and the plot from time 1-at the higher level of the latent trait-indicates that information is highest in the higher range of sores and the plot from time 7 indicates that information is highest in the lower range of scores. Both information plots, however, indicate that information is lowest in the middle range of scores. 


\section{CONCLUSION}

This study has confirmed the utility of the EdFED-I scale and indicated its strengths and weaknesses, features that are probably shared by other translations of the EdFED scale. As shown in this study - to be described in more detail elsewhere-the EdFED-I scale is a responsive indicator of change in level of feeding difficulty. While there is no apparent advantage to using a subscale score as opposed to the total score on the EdFED-I scale, it is apparent that the behavioural subscale is useful in that some of the items show IIO. That the subscale score on the invariantly ordered behavioural items — which are mostly responsive to higher levels of feeding difficulty_correlates highly with the total score supports the utility of the total score. Moreover, the observation that the putative invariance in the ordering of these items observed at baseline is sustained across this study further supports the utility of the scale. In fact, this is a unique empirical demonstration of invariant item ordering. The scale provides highest information at the lower and higher levels of feeding difficulty with a dip in information in the middle range of feeding difficulty. This indicates, for the first time, where more work is needed on the EdFED-I scale and, by implication, the EdFED scale generally; in the middle range. Ideally a scale should show the same level of information across the latent trait and that level of information should be high. Specifically, what is required is the addition of further items, which either indicate or measure feeding difficulty. It is possible, for example, that the range of feeding difficulty measured by the six behavioural items is too narrow. It is unlikely that it does not cover the latent trait at the highest level of feeding difficulty as simply allowing food to fall out of the mouth represent a very extreme level of difficulty. It is most likely that these items do not include early indicators of feeding difficulty that lie on a continuum with the existing items but which indicate a lower level of 
difficulty than, for example, spitting out food. These items remain to be discovered and some qualitative or observational work may be required to identify them.

\section{Conflicts of interest}

The Authors declare that declare that there is no conflict of interest.

They also declare that they agree with the content of this manuscript, which has not been published or submitted for publication elsewhere.

\section{REFERENCES}

1. Wimo A, Prince M. World Alzheimer Report 2010: The Global Economic Impact of Dementia. Alzheimer’s Disease International, London; 2012.

2. Prince M, Albanese E, Guerchet M, Prina M. Nutrition and dementia: a review of available research Alzheimer’s Disease International, London; 2014.

3. Watson R, Green S. Feeding and dementia: a systematic literature review J Adv Nurs. 2006;54:86-93.

4. Alzheimer's Society. Challenges facing carers of people with dementia. Alzheimer's Society, London; 2012.

5. Watson R. Practical ethical aspects of the care of patients with dementia. Nurs Ethics. $1994 ; 1: 151-162$ 
6. Prince M, Prina M, Guerchet M. World Alzheimer Report 2013: Journey of Caring - An analysis of long-term care for dementia. Alzheimer’s Disease International, London; 2013.

7. Watson R. Measuring feeding difficulty in patients with dementia: developing a scale $J$ Adv Nurs. 1994;19:257-263.

8. Watson R. Mokken scaling procedure (MSP) applied to feeding difficulty in elderly people with dementia. Int J Nurs Stud. 1996;33:385-393.

9. Watson R, Green SM, Legg L. The Edinburgh Feeding Evaluation in Dementia Scale \#2 (EdFED \#2): convergent and discriminant validity. Clin Eff Nurs. 2001;5:44-46.

10. Lin LC, Watson R, Lee YC, Chou YC, Wu SC. Edinburgh Feeding Evaluation in Dementia (EdFED) scale: cross-cultural validation of the Chinese version. $J$ Adv Nurs. 2008;62:116-123.

11. Bagnasco A, Watson R, Zanini M, Rosa F, Rocco G, Sasso L. Preliminary testing using Mokken scaling of an Italian version of the Edinburgh Feeding Evaluation in Dementia (EdFED-I) scale. Appl Nurs Res. 2015;28:391-396.

12. Meijer RR, Sijtsma K, Smid NG. Theoretical and empirical comparison of the Mokken and Rasch approach to IRT. Appl Psychol Meas. 1990;14:283-98. 
13. Watson R, van der Ark LA, Lin LC, Fieo R, Deary IJ, Meijer RR. Item response theory: How Mokken scaling can be used in clinical practice. J Clin Nurs. 2012;21:2736-2746.

14. Mokken RJ, Lewis C. A nonparametric approach to the analysis of dichotomous item responses Appl Psychol Meas. 1982;6:417-30.

15. Ramsay JO. TestGraf: a program for the graphical analysis of multiple choice test and questionnaire data. McGill University, Montreal; 2000.

16. Van der Ark L. New developments in Mokken scale analysis in R. J Stat Softw. 2012;48:5. Doi:10.18367/jss.v048.i05.

17. Kuijpers RE, van der Ark LA, Croon MA. Standard errors and confidence intervals for scalability coefficients in Mokken scaling analysis using marginal models. Sociol Methods. 2013;43:42-69.

18. Baker FB. Basics of item response theory. Eric Clearing House of Assessment and Education, University of Maryland, College Park; 2001.

19. Watson R, Deary IJ. Feeding difficulty in elderly people with dementia: confirmatory factor analysis. Int J Nurs Stud. 1997;34:405-414. 


\section{Table 1}

Number of significant violations of IIO and effect on Htrans $\left(H^{T}\right)$ in The EdFED-I Scale with sequential item removal steps $(N=401)$

Item Label

$1 \quad$ Does the patient require close supervision while feeding?

2 Does the patient require physical help with feeding?

3 Is there spillage while feeding?

$4 \quad$ Does the patient tend to leave food on the plate at the end of a meal?

5 Does the patient ever refuse to eat?

6 Does the patient turn his/her head away while being fed?

7 Does the patient ever refuse to open his/her mouth?

8 Does the patient spit out his/her food?

9 Does the patient leave his/her mouth open allowing food to drop out?

10 Does the patient refuse to swallow?
Step 1 Step 2 Step 3 Step 4 Step 5

$\begin{array}{lllll}0 & 0 & 0 & 0 & 0 \\ 0 & 0 & 0 & 0 & 0 \\ 5 & - & - & - & - \\ 2 & 2 & 1 & 1 & - \\ 2 & 2 & - & - & - \\ 4 & 3 & 2 & - & - \\ 2 & 1 & 1 & 0 & 0 \\ 2 & 1 & 1 & 0 & 0 \\ 2 & 1 & 0 & 0 & 0 \\ 3 & 2 & 1 & 1 & 0 \\ .80 & .81 & .88 & .88 & .94\end{array}$




\section{Table 2}

\section{Mean item scores for EdFED-I items across the study}

Item Label Time $\begin{array}{lllllll}1 & 2 & 3 & 4 & 5 & 6 & 7\end{array}$

1 Does the patient require close supervision while feeding?

$\begin{array}{lllllll}1.85 & 1.85 & 1.85 & 1.85 & 1.85 & 1.85 & 1.83\end{array}$

2 Does the patient require physical help with feeding?

$\begin{array}{lllllll}1.75 & 1.73 & 1.73 & 1.72 & 1.72 & 1.71 & 1.68\end{array}$

$3 \quad$ Is there spillage while feeding?

4 Does the patient tend to leave food on the plate at the end of a meal?

$\begin{array}{lllllll}0.29^{\dagger} & 0.28^{\dagger} & 0.27^{\dagger} & 0.25 & 0.26^{\dagger} & 0.26^{\dagger} & 0.24^{\dagger}\end{array}$

$5 \quad$ I Does the patient ever refuse to eat?

$\begin{array}{lllllll}0.89 & 0.80 & 073 & 0.69 & 0.67 & 0.67 & 0.64\end{array}$

6 Does the patient turn his/her head away while being fed?

$0.98^{* \dagger} 0.92{ }^{* \dagger} 0.85^{* \dagger} 0.83^{* \dagger} 0.80^{* \dagger} 0.78^{\dagger} 0.74^{\dagger}$

7 Does the patient ever refuse to open his/her mouth?

$\begin{array}{lllllll}0.28 & 0.24 & 0.23 & 0.22 & 0.24 & 0.24 * & 0.23\end{array}$

8 Does the patient spit out his/her food?

$0.34^{\ddagger} \quad 0.29^{\ddagger} \quad 0.26^{\ddagger} \quad 0.25^{\ddagger} \quad 0.24^{\ddagger} \quad 0.24^{\ddagger} \quad 0.23^{* \ddagger}$

9 Does the patient leave his/her mouth open allowing food to drop out?

$\begin{array}{llllllll}0.41^{\ddagger} & 0.35^{\ddagger} & 0.31^{\ddagger} & 0.30^{\ddagger} & 0.28^{\ddagger} & 0.27^{\ddagger} & 0.26^{\ddagger}\end{array}$

10 Does the patient refuse to swallow?

$\begin{array}{lllllll}0.28^{\ddagger} & 0.24^{\ddagger} & 0.21^{\ddagger} & 0.21^{\ddagger} & 0.21^{\ddagger} & 0.22^{\ddagger} & 0.20^{\ddagger}\end{array}$

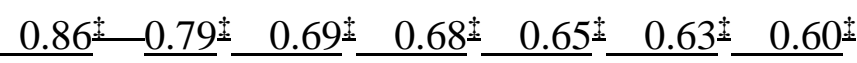

\begin{tabular}{llllllll}
$N$ & 401 & 401 & 399 & 401 & 345 & 344 & 343 \\
$H s$ & 0.46 & 0.46 & 0.47 & 0.48 & 0.48 & 0.46 & 0.48 \\
$\underline{H}^{\underline{T}}$ & 0.58 & 0.58 & 0.53 & 0.53 & 0.53 & 0.53 & 0.53 \\
\hline
\end{tabular}

Hs = Scale H; $H^{T}=$ Htrans for items 7, 8, 9, \& 10; * = items which do not scale; $\dagger=$ items where the 95\% confidence interval does not include 0.30 ; $\ddagger=$ items showing invariant item ordering 